\title{
Design Space for Driver-based Automotive User Interfaces
}

\author{
Dagmar Kern, Albrecht Schmidt \\ Pervasive Computing and User Interface Engineering Group \\ University of Duisburg-Essen, \\ Essen, Germany \\ \{dagmar.kern, albrecht.schmidt\}@uni-due.de
}

\begin{abstract}
Over the last 100 years it has become much easier to operate a car. However in recent years the number of functions a user can control while driving has greatly increased. Infotainment, entertainment and comfort systems as well as driver assistance contribute to this trend. Interaction with these systems plays an important role, as on one hand this can improve the user experience while driving but on the other hand it may distract from the primary task of driving. User interfaces in cars differ regarding the number of input and output devices and their placement in the car to a great extent. In this paper, we introduce a first design space for driver-based automotive user interfaces that allows a comprehensive description of input and output devices in a car with regard to placement and modality. This design space is intended to provide a basis for analyzing and discussing different user interface arrangements in cars, to compare alternative user interface setups, and to identify new opportunities for interaction and placement of controls. We present a graphical representation of the design space and discuss its usage in detail based on several examples. To assess the completeness of the proposed design space we used it to classify and compare user interfaces from more than 100 cars shown at IAA2007, cars from the BMW museum, and from the A2Mac1 image database.
\end{abstract}

\section{Categories and Subject Descriptors}

H.5.2 [Information Interfaces and presentation]: User Interfaces Input devices and strategies (e.g., mouse, touchscreen), B.4.2 [Input/output and data communications]: Input/Output Devices, H.1.2 [Models and principles]: User/Machine Systems - Human factors

\section{General Terms}

Human Factors

\section{Keywords}

Design space, automotive user interfaces, car user interfaces

\section{INTRODUCTION}

Driving a car today entails a lot more than operating the pedals and steering wheel and has extended well beyond operating the primary controls. With the rapidly increasing complexity of automotive user interfaces in the last decades, drivers are now confronted with many new functions inside the car. This trend is fueled by car manufactures who, in addition to improving the

Copyright held by author(s)

AutomotiveUI'09, September 21-22, 2009, Essen, Germany

ACM 978-1-60558-571-0/09/009 safety and efficiency, e.g. by adding collision-avoidance systems, also aim to improve the comfort and entertainment opportunities within their cars [4]

Thus, a car has become more than just a means of transportation; for many people, especially with longer commutes, it is now a multifunctional living space. With the help of technologies like MP3 players, GPS navigation systems and mobile phones, people use their cars as a space for media consumption, as a personal communication center or as an inter-connected workplace. Many people spend 1 hour or more per day in their car [17] doing boring routine driving tasks on their way to work and back. To make this time more valuable and driving safe it is important to provide good user experiences inside the automobile.

The use of new functionalities inevitably increases the driver's interaction with the user interface and decreases the driver's focus on driving, which is still the primary task and should have the highest priority. This makes it important to take driver distraction [18] into considerations while designing new user interfaces for cars. Independent of which kind of functionality is introduced into the car, the associated workload level (physical, visual and mental) has to be considered for safety reasons [7]. Thus, new functionalities in cars should be as minimally distracting as possible. With the design space we provide a visual representation that allows for the designer to see how adding a new control may interfere with existing controls as they occupy the same region in the design space.

In the earlier days of the car, a one-to-one mapping from control to function was common, but with the growing number of functions inside a car, e.g. about 700 functions in a BMW series 7 [5], which are also interdependent, this is no longer possible. There is a trend in automotive systems where different functions are combined in a hierarchical menu structure, which are commonplace in graphical user interfaces for computers. Such structures require the user to search through different menus to find a desired function. This creates either visual or auditory distraction or increases the cognitive load for the task. In some cases, this is not ideal, e.g. searching for the menu function that changes the radio volume might be annoying for the driver. Thus, there is a tradeoff between how many functions are quickly accessible and how overloaded the user interface is. This trade-off can be observed in many current car interface designs.

In this paper, we introduce a design space for driver-based automotive user interfaces that provides an overview of input and output devices in cars with respect to their placement, which part of the body they interact with, which kind of feedback they provide and to which task-class they are assigned. 
For generating this design space, we analyzed 706 photographs of 117 models from 35 different manufacturers taken at the international automobile exhibition (IAA ${ }^{1}$ 2007) in Frankfurt. The photos are available at https://www.pcuie.unidue.de/AUI/IAA2007. Additionally we accessed the suitability of the design space by picking a random set of pictures from A2 $\mathrm{Mac}^{2}{ }^{2}$ image database and by modeling selected historic cars.

The central contribution of the paper is a comprehensive design space for driver-based automotive user interfaces that is grounded in an analysis of a large number of existing cars, including historic cars and concept car.

The paper is structured as follows. After discussing the background and related work, we present a graphical representation of the design space. We discuss in detail input modalities, output modalities, and position of the controls. Using two actual cars we show how the design space can be used for comparison. Additionally we show an overview representation that allows to describe a set of cars, and we show how this can be applied.

\section{BACKGROUND AND RELATED WORK}

\subsection{Driving Task}

The complex driving task can be divided into three classes primary, secondary and tertiary [16]. Primary tasks describe how to maneuver the car, e.g. controlling the speed or checking the distance to other cars or objects. Secondary tasks are functions that increase the safety for the driver, the car and the environment, e.g. setting turning signals or activating the windshield wipers. Tertiary tasks are all functions regarding entertainment and information systems.

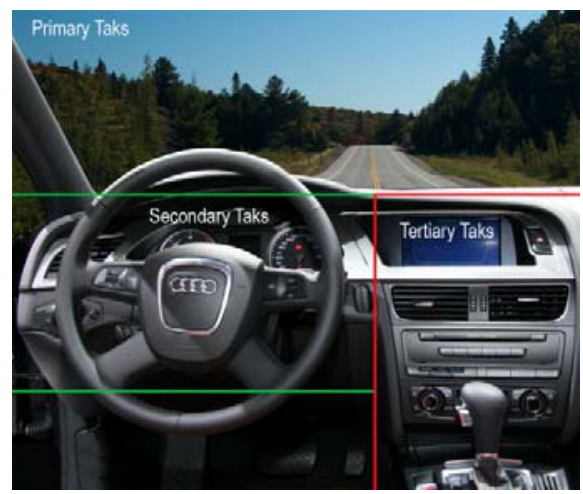

Figure 1: Distribution of primary, secondary and tertiary tasks (based on [20])

\subsection{Input Devices}

Based on the classification system from Geiser [16], Tönnis et al. [20] assigned input devices to the three classes. They distinguish between primary, secondary and tertiary devices and assign them to specific locations of the car (see Figure 1). Primary devices are used to maneuver the car, e.g. the steering wheel and the pedals. They are usually mapped one-to-one with their functionality and provide haptic feedback. Primary devices are arranged close to the driver so that they are easy to reach. Secondary devices are, for example, stalk controls for the turn signal or windscreen wipers.

\footnotetext{
${ }^{1}$ http://archiv.iaa.de/07/index.php?id=home2007\&L=1

${ }^{2}$ A2macl Automotive Benchmarking: http://a2macl.com/
}

They are also at an easy-to-reach distance, often mounted on the backside of the steering wheel. Tertiary devices are used for the info- and entertainment systems. Many manufacturers combine a large number of enter- and infotainment functionalities into one system, e.g. the Audi MMI system [2] or the BMW iDrive [6], which consists usually of two parts: a single controller and a display. Tertiary devices are often placed in the center stack. With multifunctional steering wheels, a few tertiary devices intrude into the domain of secondary devices, e.g. radio controls on the steering wheel for faster access to frequently-used functions.

\subsection{Output Devices}

Output devices are used to provide feedback to the user about the current state of the system e.g. about the current speed, if the direction indicator is turned on, or which radio channel is currently playing. Feedback is important but prioritized differently for the three different driving tasks. Feedback about the primary task must be immediate and clear, whereas the information about which radio channel is playing is less important. Output devices for providing three kinds of feedback are available in cars. They provide visual, auditory and haptic/tactile feedback or even a combination of them. A detailed discussion about issues concerning these displays can be found in [20].

\subsection{Design Guidelines and Standards}

There is a big difference in designing user interfaces for the computer domain, where the user pays full attention to the interaction, and for cars, where user's main focus has to be on the primary driving task. Interacting with tertiary user interfaces never has the highest priority for the user when the car is moving. There are international standards available $[13,14]$ that give interaction design recommendations and enforcements, e.g. the user interface must not force the user to take both hands off the steering wheel.

Furthermore, there are a few guidelines that offer support to designers during the design process, e.g. $[1,12,19]$. They describe how to make entertainment und infotainment system safe and easy to use for all drivers. They include concrete design recommendations, e.g. text size or the placement of displays always taking safety and usability issues into account.

\subsection{Design spaces}

The importance of understanding design spaces for user interfaces is emphasized by HCI researchers. Foley et al [15] provide a classification of input devices using the graphic subtask they were capable of performing. Buxton [8] introduced a taxonomy of input devices. His classification includes the physical properties and the number of spatial dimensions the devices sense. In Card et al's [9] design space, input devices are compositions of one-dimension sensors. Ballagas et al. [3] have taken up these design spaces and provide a design space of ubiquitous mobile input. In the output domain, there are also design spaces regarding structuring information visualization [10].

A design space for automotive user interfaces differs from the aforementioned design spaces in two main areas. First, all devices are fix-mounted in a car, and it is therefore essential to take placement of the devices into consideration. Second, the driver is limited in her mobility but can act with the left or the right hand, as well as with the left or right foot. 


\section{DESIGN SPACE FOR DRIVER-BASED AUTOMOTIVE USER INTERFACES}

In this section, we present our design space for automotive user interfaces, which gives a common basis to discuss existing arrangements of user interfaces in cars and aims to find new spaces for them. We focus on user interfaces that are operated by the driver, but the proposed design space can be extended to include passenger-based user interfaces. Following the view of Tönnis et al. [20], that cars are "complex computer systems with very particular input and output devices and mobile functionality", we decided to create a design space that includes all input and output devices, their connection to each other and their placement.

Our design space is based on an analysis of 706 photographs taken at the IAA 2007. We collected photographs of 117 models from 35 different manufacturers, tagged and categorized them, and looked for similarities and differences. First, we identified the different input and output modalities that can be found in almost all of the observed cars. Then, we analyzed the position and interaction model for input and output devices. The photos can be accessed at https://www.pcuie.uni-due.de/AUI/IAA2007.

The following assumptions and statements refer to left-hand cars, but they can be easily applied to right-hand cars by substituting "left" for all occurrences of "right" and "right" for "left".

\subsection{Input Modalities}

We found eight different input possibilities. The most commonly used group are buttons, which are present in different sizes and shapes. Nearly all buttons in modern cars are soft buttons (see Figure 2-a). That means there is no permanent haptic feedback available; instead, a visual feedback is often used. For example, when the high beams are turned on, this is indicated lighting up a button. In the past, mechanical buttons were used, e.g. to turn on the lights. These buttons provided haptic feedback, e.g. when a button was pressed, it felt pushed in (see Figure 2-b). Thus, the driver could determine the state of the button without looking at it. Sliders form the next group. They are often used for adjusting the direction of the fan (see Figure 2-g). We distinguish two different kind of knobs, those that are continuous (see Figure 2-d), e.g. to control radio volume, and those that are discrete (see Figure 2-c), e.g. a knob used to adjust the temperature. Stalk controls are often attached to the steering wheel to indicate or to activate windscreen wipers (see Figure 2-e). On a multifunctional steering wheel, thumbwheels are often used to control volume (see Figure 2-j). Classical pedals are still available in the car: gas, brake and (in

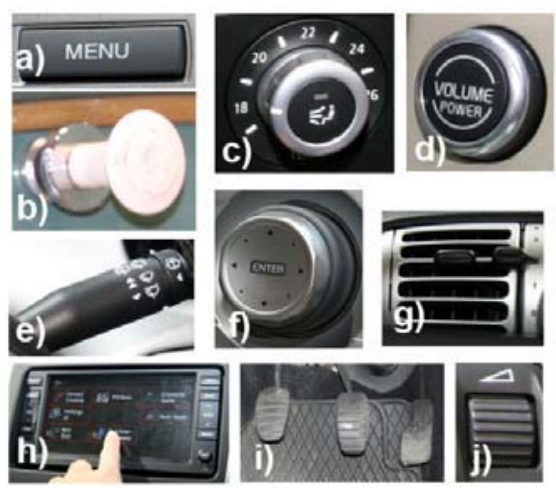

Figure 2. Input modalities: a) button b) button with haptic feedback c) discrete knob d) continuous knob e) stalk control f) multifunctional knobs g) slider h) touchscreen i) pedals j) thumbwheel. cars with stick shift) clutch (see Figure 2-i). In the last few years, more and more manufactures have added a multifunctional controller to their cars. A multifunctional controller can be turned, pressed and sometimes shifted in four or even eight directions, e.g. BMW iDrive or Audi MMI (see Figure 2-f). These controllers are combined with high-resolution displays, and together, they are used as a control unit for entertainment and infotainment systems in the car.

New interaction techniques like speech and gesture recognition, as well as indirect interaction like fatigue detection using an eye tracker or cameras, have also found their way into the car. These new interaction techniques provide means for hands-free interaction so that drivers no longer need to search for and touch specific devices while driving. However, speech recognition often requires the driver to push a push-to-talk button before it can be used.

Touchscreens, the last input opportunity, are at the border to the output modalities, because they combine both input and output modalities in a single device (see Figure 2-h). The application areas for touchscreens are enter- and infotainment systems as well as comfort systems like air-conditioning systems.

\subsection{Output Modalities}

The output modalities are limited by the human senses, specifically sight, hearing, touch and smell. There are a lot of visual indications available in the car to give feedback about current functional states. These indications vary from simple indicator lamps to high-resolution displays. Looking closer at the simple indicator lamps, e.g. those used to indicate that the high beams are turned on, you can find two different ways to present information. One way is to turn on a light above a description (see Figure 3-d), and the other way is to illuminate a symbol whose shape indicates the meaning (see Figure 3-e).

Visual representations are also used to give information that is directly correlated to the driving task, e.g. actual speed. Both analog and digital representation are used for these purposes (see Figure 3, a-b). Analog representations can also be divided into displays that use a physical dial and pointer and displays that replicate the dial and pointer virtually (see Figure 3-c). Virtual representations allow for more dynamic use of the space in the middle of the dial to show other information. Digital displays have been used since the end of the 1970s to show alphanumerical information, e.g. the current radio channel or traffic information (see Figure 3-g).
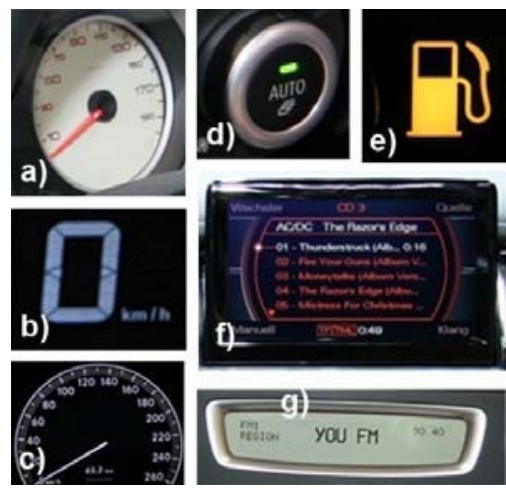

Figure 3. Output modalities: a) analog speedometer b) digital speedometer c) virtual analog speedometer d) indicator lamp e) shaped indicator lamp f) multifunctional display g) digital display 


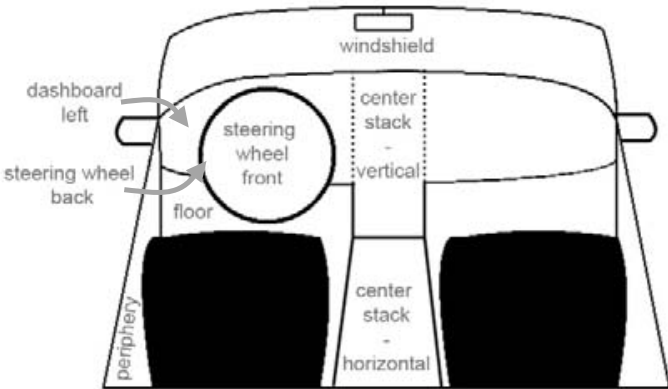

Figure 4. Division of driver's interaction environment.

At the end of 1990s, multifunctional LCT or TFT displays started to appear in cars, and car manufactures started to integrate comfort, entertainment and infotainment functions into single systems. These systems are controlled by buttons on each side of the screen, by a central controller or by touchscreen.

Sense of hearing is addressed by loudspeakers, which are integrated into the car or attached to an external device, e.g. a portable navigation system. This modality has long been used for entertainment purposes and has more recently been used for giving aural feedback, especially with voice-operated systems.

Information can also be delivered to the driver by using the sense of feel or touch. Some car manufactures have recently added vibration feedback to the steering wheel or to the driver's seat to warn the driver, e.g. of lane departures when no turn indication has been made [11]. In the earlier days, cars already relied on sense of touch with mechanical buttons whose physical state gave direct feedback.

Output modalities that use the sense of smell have yet to be established. However, one can imagine that this sense could be used for more ambient information. For example, when the motor temperature is increasing, the odor inside the car could change.

\subsection{Positioning Input and Output Devices}

The arrangement of input devices in cars is limited by ergonomic factors. All input devices have to be within reach for the driver, so that she can safely manipulate them with the left or right hand or left or right foot while driving. Except for touchscreens, output devices do not necessarily have to be within a safe reaching distance, but they do need to be in the driver's field-of-view.

We identified the following main interaction areas between the driver and the car (see Figure 4):

- Windshield: used for example for head-up displays

- Dashboard: for driver-based user interfaces we focus on the left part of the dashboard that is directly in front of the driver in left-hand cars.

- Center Stack: divided into the vertical part (on the right side of "dashboard left" in front of the driver) and the horizontal part (between the front seats)

- Steering wheel: divided into front and back side of the steering wheel

Floor

- Periphery: includes the side-/rear-view mirrors

\subsection{Graphical Representation}

We propose two different graphical representations, one for categorizing a single car and the other for analyzing a set of cars that can be used for comparing cars from different manufacturers or car models from different years.

\subsubsection{Categorizing a single car}

In our two-dimensional graphical representation, we focus on the placement and the task classification of input and output devices based on what body part would interacts with them. We regard the driver as the main user and create the interaction descriptions from the driver's point of view.

The first dimension of the graphical representation indicates the placement of devices: windshield, dashboard (left), center stack, steering wheel, floor, and periphery. The other dimension is given by input and output modalities, where input is divided into left or right (hand or foot) as the main interaction initiators. We added one more column for input devices to represent additional modalities like speech to the design space. Since the voice has no direct spatial representation, it is associated with the periphery area. The output modalities are divided into the three senses: sight, hearing, and haptic (for feel and touch). If new interaction methods cannot be located in the current dimensions, a new column can be introduced, e.g. gesture as input or airflow/olfactory as output, to represent a new modality.

Each input or output device can be added into the grid shown in Figure 5. The symbolic representation of different device types allows the design space to be extended with new modalities. For example, a sensor to measure skin conductivity that is mounted on the steering wheel would be represented by a new symbol and placed in the section representing the steering wheel. The structure of the design space would remain the same, allowing the new modality to be compared with the others without limiting the design space to the current set of modalities.

We divided the devices into the three task categories defined by [20], primary, secondary and tertiary tasks. These categories are color-coded in the graphical representation. Info- and entertainment systems, as well as comfort functions like air conditioning, could be clearly classified as tertiary tasks, but driver assistance systems like Adaptive Cruise Control are not so easy to classify. Tönnis et al. [20] suggested classifying them as secondary-task devices, but we believe they are rather used for primary tasks, because they influence the driving task directly.

Numbers inside the symbols indicate the occurrences. Dotted lines illustrate connections between input and output devices. Lines ending with arrows represent direct connections (e.g. stalk control for headlight gives visual feedback with indicator lamp) and ending with dots represent indirect connections to an output domain (e.g. volume knob controls audio volume and gives no localized feedback). Numbers on the lines indicate how many controls are connected to the output devices/domain.

In Figure 5, two graphical representations for a 2007 BMW 520d and a 1956 BMW 507 are shown. Each input and output device is classified in the graphical representations. Classifying the head-up display was a unique case, since it fits into all three task categories. Thus, its display-symbol is divided into three parts, one for each task. Section 4 contains discussion comparing the two cars based on these classification results. 

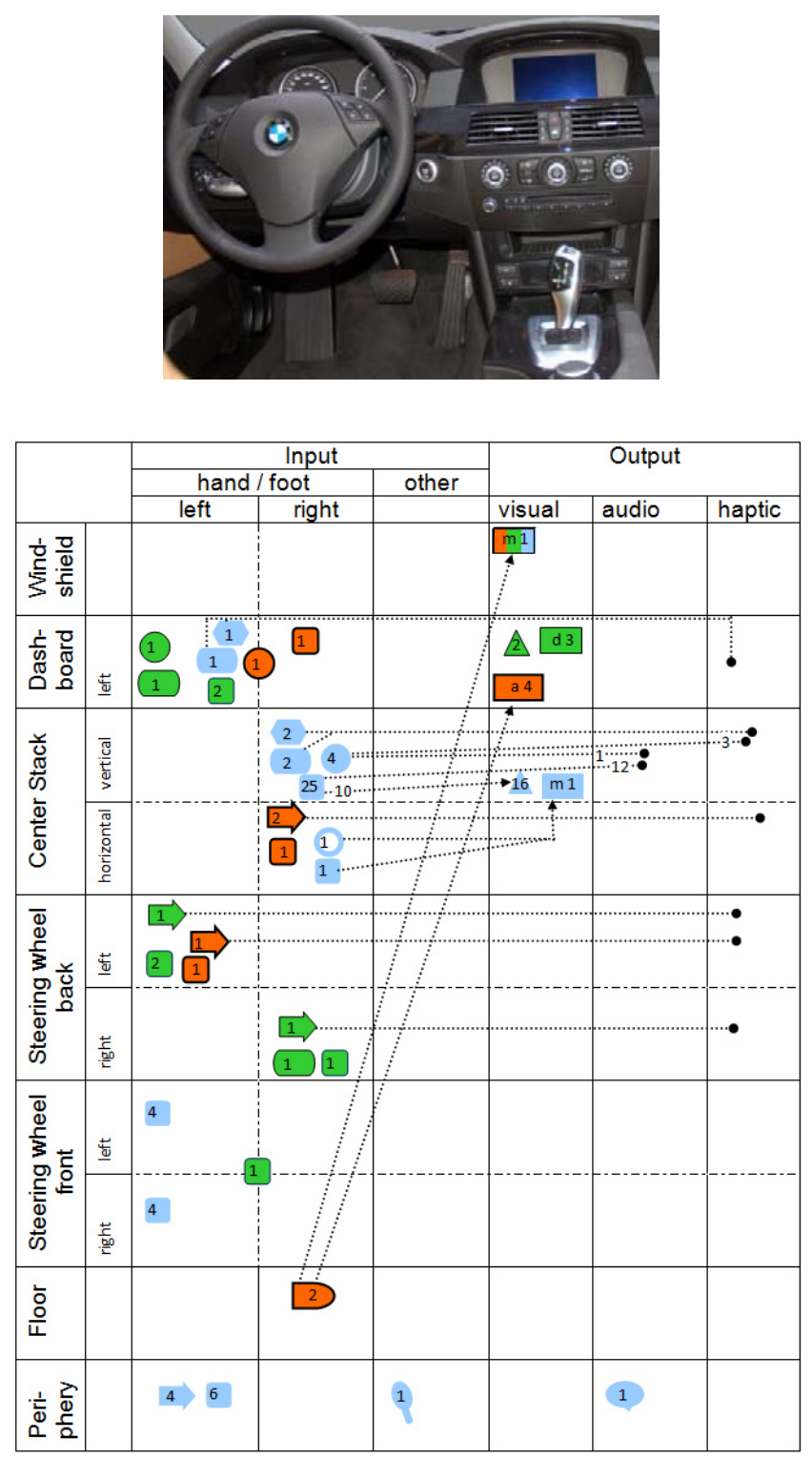
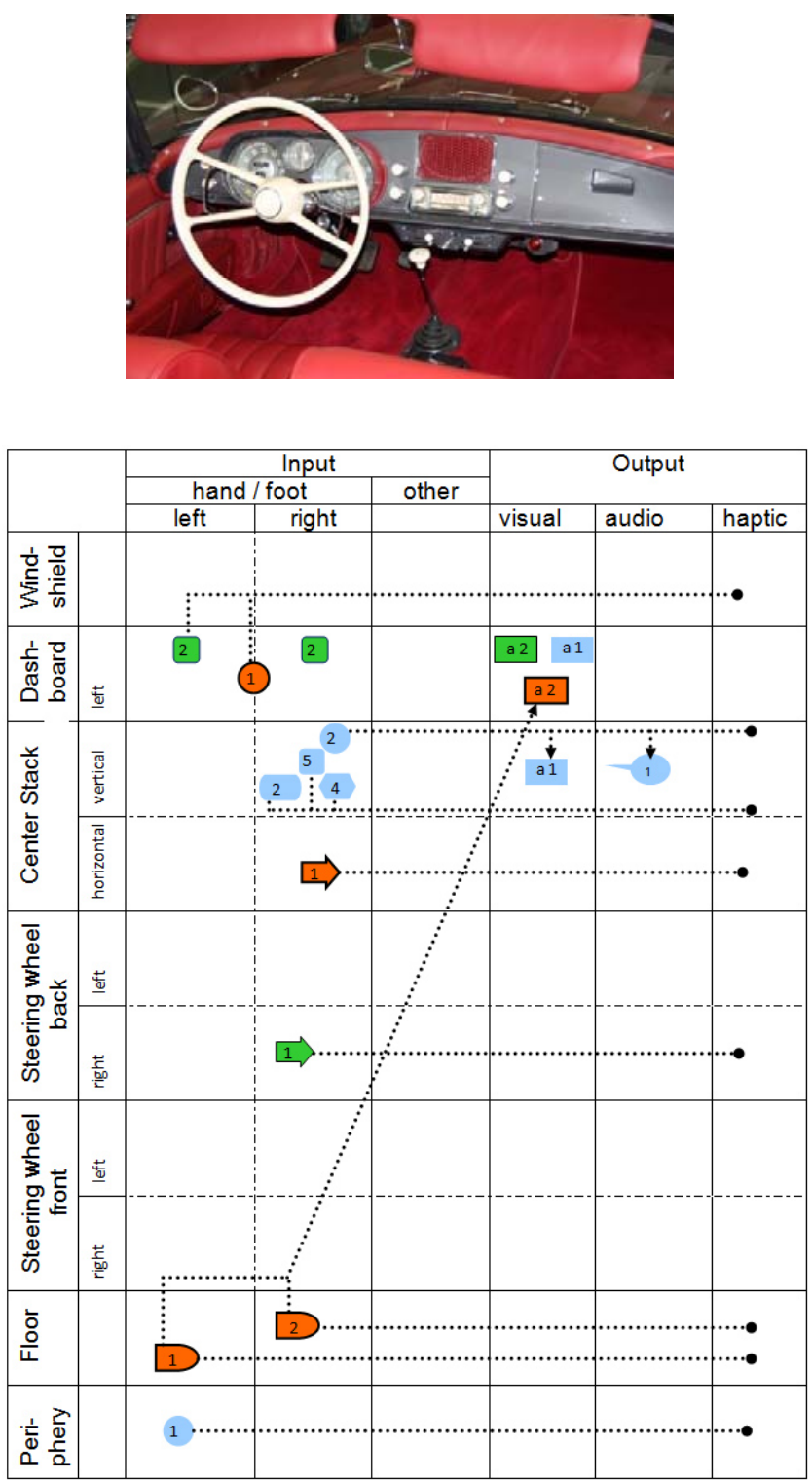

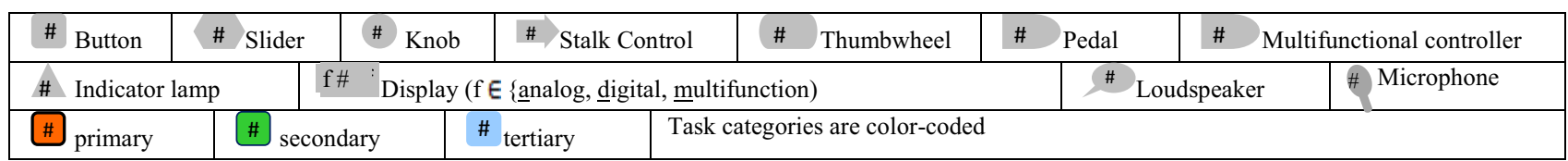

Figure 5. Graphical representation of our design space for driver-based automotive user interfaces. The classifications were created for a 2007 BMW 5 series and a 1956 BMW 5 series. Both cars have the steering wheel on the left side. The design space consists of the different interaction areas in a car, to which the input modalities are assigned. Output is divided into visual, audio and haptic. Numbers inside the controls indicate the occurrences. Primary, secondary and tertiary tasks are color-coded. 

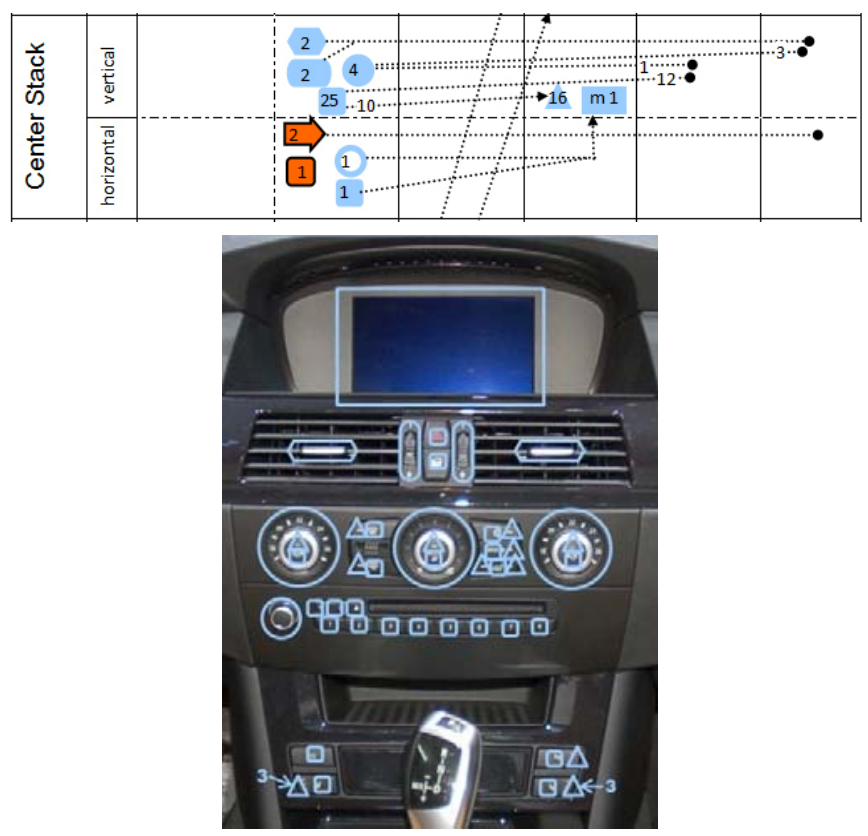

Figure 6. Detailed view of the center stack area. Corresponding markers (vertical) are shown in the photograph.

Figure 6 illustrates the connection between the symbols used in the graphical representation to real devices for the BMW 520d. In the photograph, the devices are marked by the same symbols. In this BMW series 5, 25 buttons are available, from which 10 provide visual feedback with an indicator light, and 12 are associated with the radio and provide audio feedback. The remaining 3 buttons influence the air conditioning system but provide no direct feedback. The two sliders and thumbwheels provide haptic feedback through their current positions. One continuous knob is used to control the volume, and the other three discrete knobs control the air conditioning system and provide visual feedback by indicating at which temperature they are set. The LCD screen shows visual feedback and is controlled by the iDrive controller, which is mounted in the center stack. Each of these input devices can be specified further using Card et al.'s design space for input devices [9].

This center stack example further illustrates that it is possible to analyze a select part of the design space. Still, it must be taken into account that some input-output connections may get lost.

\subsection{Analyzing a Set of Cars}

For providing a more general view, an abstract representation of the design space is illustrated in Table 1 and 2. The areas are not separated into subareas but instead represented by a triple, which stands for (primary, secondary, tertiary). This abstraction can be used to categories a set of cars, as in Table 1 with different BMWs or Table 2 with different Renault cars.

Analyzing the abstract views of the design space classifications, we found that the BMW models were all very similar in their arrangement of input and output devices, while Renault offered a wider selection of arrangements, especially with devices for tertiary tasks (e.g. the number of controls on the vertical center stack that can be controlled by the driver's right hand in the range 6 to 41). From these abstract views, similarities and differences can be extracted. For example, the floor and horizontal center stack areas are very similar. The number of devices in the floor area only differ for automatic or manualtransmission cars, which was the same for both manufacturers. The variation in the numbers of devices correlated to the number of their functionalities. For example, additional devices for Adaptive Cruise Control (ACC) were needed or a multifunctional controller was used for models have with a multifunctional display.

\begin{tabular}{|c|c|c|c|c|}
\hline & \multicolumn{3}{|c|}{ Input } & Output \\
\hline & left & & right & \\
\hline Windshield & & & & $(0,1,1)$ \\
\hline Dashboard & $(0,4,2)$ & $(1,0,0)$ & $(1,0,0)$ & $(4,2-5,0)$ \\
\hline Center stack & & & $(3,0,35-50)$ & $(0,0,17-20)$ \\
\hline $\begin{array}{l}\text { Steering } \\
\text { wheel (back) }\end{array}$ & $(0-2,3-5,0)$ & & $(0,3,0)$ & \\
\hline $\begin{array}{l}\text { Steering } \\
\text { wheel (front) }\end{array}$ & $(0,0,4)$ & $(0,1,0)$ & $(0,0,4)$ & \\
\hline Floor & $(0-1,0,0)$ & & $(2,0,0)$ & \\
\hline Periphery & & $(0,0,11)$ & & $\begin{array}{l}(0,0,3-4) \\
\end{array}$ \\
\hline
\end{tabular}

Table 1. Classification of BMW models series $1,3,5$ and M3

\begin{tabular}{|c|c|c|c|c|}
\hline & \multicolumn{3}{|c|}{ Input } & Output \\
\hline & left & & right & \\
\hline Windshield & & & & $(0,0,2)$ \\
\hline Dashboard & $(0,2-4,2-11)$ & $(1,0,0)$ & $(0-2,0-2,0)$ & $(0-4,0-3,0)$ \\
\hline Center Stack & & & $(2,0,6-41)$ & $(0-4,0-3,1-18)$ \\
\hline $\begin{array}{l}\text { Steering } \\
\text { wheel (back) }\end{array}$ & $(0,3,0-4)$ & & $(0,5,4)$ & \\
\hline $\begin{array}{l}\text { Steering } \\
\text { wheel (front) }\end{array}$ & $(0-2,0,0)$ & $(0,1,0)$ & $(0,0,0-2)$ & \\
\hline Floor & $(0-1,0-1 ; 0)$ & & $(2,0,0)$ & \\
\hline Periphery & & $(0,0,8)$ & & $(0,0,3-5)$ \\
\hline
\end{tabular}

Table 2. Classification of Renault models Clio, Espace, Kangoo, Koloes, Laguna, Megane, Modus, Twingo.

\section{USING THE DESIGN SPACE}

\subsection{Historical Analysis and Trends}

Our proposed design space can be used to analyze trends and explore historical changes. Regarding historical changes, we found that few controls stay where they were, especially for control of primary tasks. Primary-task controls have not changed at all in the last years (e.g. steering wheel or pedals). The trend towards automatic-transmission cars decreased the pedals to two. Another trend, towards facilitating the driver while driving, leads to an increase of devices for primary tasks e.g. for (Adaptive) Cruise Control. In the secondary task domain, there is a trend away from analog speedometers towards digital speedometers in both discrete and continuous types. Some manufactures also changed the position of the visual output of the speed from the driver's side to the middle.

A huge increase in the number of devices for tertiary tasks can also be observed, which is strongly related to the increase in comfort, entertainment and infotainment functionalities in cars 
e.g. air conditioning, integrated support for mobile phones, navigation systems, and MP3 players.

Figure 5 clearly illustrates the difference in the number of devices. The 2007 BMW series 5 has 113 devices (13 primary, 15 secondary, 85 tertiary), resulting in the input triple $80(9,11$, 60 ) and output triple $33(4,4,25)$. In contrast, the 1954 BMW series 5 has 29 devices (7 primary, 9 secondary, 13 tertiary), with input triple $21(5,5,11)$ and output triple $8(2,4,2)$. Another big difference can be seen in the feedback opportunities of the buttons. All buttons in the 1954 car has haptic feedback while buttons in the 2007 car has visual feedback. Furthermore, the steering wheel area is becoming more important. Whereas the 1954 BMW only has secondary controls mounted on the back of the steering wheel, the 2007 BMW has controls for all three task classes on the front and back of the steering wheel.

\subsection{Analysis of IAA2007}

Using our proposed design space, we were also able to analyze the photographs taken at IAA 2007 in more detail.

One trend that we found is that the space on the steering wheel is often used for controls, e.g. for hands-free interaction with mobile phones or controlling the entertainment system. $78 \%$ of the cars have controls on the steering wheel. Another trend is the use of displays in cars for navigation systems and other comfort functionalities. $72 \%$ of the researched cars already have a builtin display. Display types are evenly balanced between touchscreens and non-touchscreens (46\% have a touchscreen). Touchscreens are mostly found in American and Japanese cars, while German cars almost exclusively followed the concept "display controlled with controller".

An indication of future trends could also be seen in the presented concept cars. Citroen, for instance, has a display and the main controls on the steering wheel in their concept car "Cactus", In general, we observed that the display space in concept cars is much bigger than in current cars. Displays for front-seat passengers are also prevalent.

\subsection{Looking for New Ideas}

With the introduction of automatic-transmission cars, the clutch pedal disappeared, freeing up space for other controls. It would be interesting to see if the left foot could be used for interaction with controls in this space, e.g. for zooming in/out in a navigation system. Currently, input modalities on the steering wheel consist of buttons and thumbwheels. The Citroen concept car Cactus, however, already has a display mounted on the steering wheel. It might be interesting to look more into new input and output opportunities on a steering wheel. Handwriting input on a steering wheel, for example, may be easier than in the center stack for left-handed people in cars with the steering wheel on the left side or for right-handed people in a car with the steering wheel on the right side.

With head-up displays, the windshield is also becoming an important new area for output modalities. In addition to providing visual feedback for systems, the windshield area may also hold opportunities for spatial audio.

The front-seat passenger area also provides open space that is not directly represented in the design space, because it can't be concretely used by the driver, but one can imagine having an

\footnotetext{
${ }^{3}$ http://wikicars.org/en/Citro\%C3\%ABn_Cactus-C
}

additional screen there where the passenger can interact with incar systems, e.g. enter entries in the navigation system, and send the results to the driver's screen.

It is also visible from the design space that new modalities (e.g. haptics) can find spaces that are not yet occupied by other controls.

\section{CONCLUSION}

In this paper, we presented a design space for driver-based automotive user interfaces with respect to the placement of devices in the car and the body parts that interacts with these devices. Our design space is based on an analysis of photographs taken from 117 different cars from 35 manufacturers. We discussed different input and output modalities in cars and presented a graphical representation for categorizing individual cars that should help user interface designers analyze existing layouts, generate new ideas, and find unexplored areas for future designs. Furthermore, we provide a more abstract graphical representation for comparing a set of cars to find concrete similarities and differences between different manufactures or different types of cars, e.g. comparing middle-class and luxury cars.

The design space is based on the analysis of left-hand cars but it can be used for right-hand cars as well. When comparing cars, it is easiest to analyze only one type of car (left or right handed) with this design space. Cross-comparisons are also possible but require changing left and right columns for the dashboard and the center stack for either the right-hand or left-hand cars.

We discussed the usage of the design space by looking at historical changes and trends as well as differences between the 117 cars based on photographs taken at IAA2007.

We showed that this design space can be used as a tool for comparing different user interface options and layouts as well as a means to facilitate a structure discussion of existing and future car user interfaces.

In the future we plan to include additional measure, possibly automatic, that detect potential design flaws that would impact driver performance. We envision a software tool that assists designers in choosing and placing controls into the design space. This software tool might allow the designer to mark specific controls in a picture or in a design sketch, from which a graphical representation could automatically be generated. The tool might also provide immediate estimated feedback on the impact of the control placement on the driver, e.g. with regard to visual load or cognitive load.

Both graphical representations shown in Fig. 5 can be found at https://www.pcuie.uni-due.de/AUI/. This wiki can be used to exchange design spaces with others. The design spaces are ordered by label and year of construction. Additionally, a design space template is available at https://www.pcuie.unidue.de/AUI/.

\section{ACKNOWLEDGEMENTS}

This work was performed in the context of the DFG (Deutsche Forschungsgemeinschaft) funded research group 'Embedded Interaction'.

\section{REFERENCES}

[1] Alliance of Automobile Manufacturers: Statement of Principles, Criteria and Verification Procedures on Driver 
Interaction with Advanced In-Vehicle Information and Communication Systems, 2003.

[2] Audi glossary MMI-Multi Media Interface: http://www.audi.co.uk/audi/uk/en2/tools/glossary/operation comfort/MMI Multi Media Interface.html

[3] Ballagas, R., Rohs, M., Sheridan, J., Borchers, J., The Design Space of Ubiquitous Mobile Input. In Joanna Lumsden, editor, Handbook of Research on User Interface Design and Evaluation for Mobile Technologies. IGI Global, Hershey, PA, USA, 2008.

[4] Bishop, R, Intelligent vehicle technology and trends. London: Artech House Publishers, 2005.

[5] BMW 7 Series BuisnessWeek http://www.businessweek.com/lifestyle/content/sep2008/b w2008099 699585 page $2 . \mathrm{htm}$

[6] BMW Technology Guide iDrive: http://www.bmw.com/com/en/insights/technology/technolo gy_guide/articles/idrive.html

[7] Burnett, G.E, Designing and evaluating in-car userinterfaces. In J. Lumsden (Ed.) Handbook of Research on User-Interface Design and Evaluation for Mobile Technology, Idea Group Inc. 2008.

[8] Buxton, W., Lexical and pragmatic considerations of input structures. SIGGRAPH Comput. Graph., 17(1), 31-37, 1983.

[9] Card, S. K., Mackinlay, J. D., Robertson, G. G., A morphological analysis of the design space of input devices. ACM Trans. Inf. Syst., 9(2), 99-122, 1991.

[10] Card, S. K., Mackinlay, J., The structure of the information visualization design space, Proceedings of the 1997 IEEE Symposium on Information Visualization (InfoVis '97), p.92, October 18-25, 1997

[11] Citroen UK Technology - Safety - Lane Depature Warning System: http://www.citroen.co.uk/technology/safety/ldws/
[12] Commission of the European Communities: Commission Recommendation of 22 December 2006 on safe and efficient in-vehicle information and communication systems: Update of the European Statement of Principles on human machine interface, 2006.

[13] DIN Deutsches Institut für Normung. Road vehicles Ergonomic aspects of transport information and control systems - Dialogue management principles and compliance procedures, Mar. 2003. Ref.No.: DIN EN ISO 15005:2003.

[14] DIN Deutsches Institut für Normung. Road vehicles Ergonomic aspects of transport information and control systems - Specifications and compliance procedures for invehicle visual presentation, Oct. 2003. Ref.No.: DIN EN ISO 15008:2003-10.

[15] Foley, J.D., Wallace, V.L., Chan, P., The human factors of computer graphics interaction techniques. IEEE Comput. Graph. Appl. 4, 11 (Nov 1984), 13-48.

[16] Geiser, G. Man Machine Interaction in Vehicles. ATZ 87, 1985. Pages 74 - 77.

[17] National Household Travel Survey: Commuting for Life, November 2006, http://nhts.ornl.gov/briefs/Commuting\%20for\%20Life.pdf

[18] Pettitt, M.A., Burnett, G.E. and Stevens, A. Defining driver distraction. Proc. World Congress on Intelligent Transport Systems, 2005

[19] Stevens, A., Quimby, A., Board, A., Kersloot, T. and Burns, P. (2002). Design guidelines for safety of in-vehicle information systems. Project report PA3721/01, TRL Limited.

[20] Tönnis, M., Broy, V., and Klinker, G.. A Survey of Challenges Related to the Design of 3D User Interfaces for Car Drivers. In Proceedings of the 1st IEEE Symposium on 3D User Interfaces (3D UI), March 2006. 